\title{
Health Care Informatics
}

\author{
Ghada Ahmed Hassan \\ lecturer of pediatric, Menoufia University \\ ghada_ahmed1982@yahoo.com
}

\section{Abstract}

Health informatics (also called Health Information Systems) uses information technology to organize and analyze health records to improve healthcare outcomes. Health Informatics deals with the resources, devices and methods to utilize acquisition, storage, retrieval, and use of information in health and medicine. Tools include medical terminology, information and communication systems and computer technology. Healthcare informatics provides electronic access to medical records for patients, doctors, nurses, hospital administrators, insurance companies and heath information technicians. The Health Informatics field is rapidly growing and there are many online and on-campus certificate and degree programs in health informatics and health information management at both the undergraduate and graduate levels.

\section{Introduction:}

In health sciences there are procedures of high complexity with reference to biological organizations \& their functions. A disease is very often a result of interactions of a patient in their environmental and psychosocial context. Therefore, when evaluating health, many considerations should be made, and a large number attributes need to be collected, stored and retrieved during decision making. The information is produced and communicated during the above mentioned procedures: there are multiple users that share this information in a hospital and outside of the hospital. Very often, same data 
are accessed concurrently by different health providers, who will need this data for different purposes. Information changes dynamically during the care provision, for example when a new exam is prescribed or when the treatment plan of a patient is altered. It is also important to understand that the health care information derives from the combination of many atomic health data, which have to be assessed together to produce useful information for the clinical decision making. The majority of health related information coming from the analysis of numerical, Boolean data but also from images, video and sound data. Definitions of Health Informatics:

\section{World Health Organization Definition:}

'an umbrella term referring to the application of the methodologies and techniques of information science, computing, networking and communications to support health and health related disciplines such as medicine, nursing, pharmacy and dentistry'

\section{Edward H. Shortleaf Definition:}

The field that concerns itself with the cognitive, information processing, and communication tools of medical practice, education and research Including the information science and the technology to support these tasks'

Health Informatics is therefore an intersection of information science, computer science, and health care. It studies the resources, devices and methods required, in order to optimize the:

- Acquisition

- Storage

- Retrieval

- Use of information in health. 


\section{Why Health Informatics?}

- Health Informatics provides information to make decisions

- Better information leads to better decisions

- Health care, management, planning and policy all need good information

- Health care, health management, health policy and health planning all depend on having good information to make decisions.

\section{Categories of Information in Healthcare}

In terms of the scope of the use of information, there are four categories of health care information: clinical, administrative, financial and population health information, all used in various ways to facilitate decision making. There are some important considerations related to health care data: Information is data that have meaning: It can be presented in any medium (text, lists or graphics) in the manner that the end user prefers.

\section{Methods of utilizing data to inform clinical decisions}

A health professional utilizes four critical methods involving data, to make decisions:

(i) Medical knowledge base

(ii) Information coming from patient

(iii) Experience and judgment.

(iv) Application of data mining methods on historical data for knowledge acquisition.

The medical knowledge base: Is acquired via studying, revisiting, reviewing the medical knowledge of a health professional's area of expertise. It is not limited to the university acquired knowledge, but also involves continuous 
education, reviewing recent literature and publications, attending conferences and scientific meetings.

Information coming from patient: includes and is not limited to, the patient history, medical exams, vital signs, radiology tests.

Experience, medical judgment: we refer to the clinician putting together all the different pieces of a puzzle, including the three components above, to decide on a diagnosis for a patient and consequently which one would be the treatment options. Doctors, during the medical judgment have learned how to do a differential diagnosis, which is the process of differentiating between two or more conditions that share similar signs or symptoms. For nurses, the nursing assessment is part of the nursing process and is a systematic procedure that nurses follow. It involves the collection and analysis of the available patient data, and is not limited to the physiological, but also involving psychological, sociocultural, and lifestyle factors too

Data mining for knowledge acquisition: algorithms are applied on large datasets to predict health related events for a patient, like the diagnosis, the prognosis, and the optimal treatment plan. This component is the most recent addition to the clinical decision making mechanism, and still remains unexplored or, at best, underutilized in most hospitals. These systems should serve as an additional input for the health professional, who in their turn, will be expected to use this extra input for more accurate and less error prone medical decisions. It is generally agreed, in the literature that these systems can neither act autonomously, nor can dictate to the health professional, the appropriate practices. Decision-making systems, on the contrary, should be designed as an extension to the human cognitive process that takes place during decision making 


\section{Key Elements of Informatics Acquisition:}

Capture data produced during health care provision. There are different ways that this is achieved in a clinical environment:

1. Observation and clinical examination: doctors and nurses collect crucial information about patients using the medical and nursing evaluation procedures, which include systematic steps to assess a patient condition from a medical and nursing perspective. Doctors also perform the clinical examination which includes palpation, inspection, percussion and auscultation. This way, doctors use their natural senses and tools which amplify our natural senses abilities while examining a patient.

2. Talking with the patient, talking with other health professionals. Especially the interactions between nurses and doctors are of great importance, taking into consideration that nurses spend a considerable amount of time with the patient and are able to do nursing observations which can provide an invaluable input to the doctor. A nurse can typically see small changes or events to a patient's condition, like for example loss of appetite, skin colour changes, change of consciousness. for example measuring how well the heart is functioning by taking an ECG (electrocardiograph.). Typically, the majority of physiological measurements are performed by nurse practitioners.

4. Laboratory tests and radiology examinations: laboratory tests involve the analysis of samples extracted from patients (i.e. blood, urine, tissue). Typically, a laboratory test is a part of regular check-up, but during a patient hospitalization, these are usually performed too. 